\title{
Suitability of Tunneling Ionization Produced Plasmas for the Plasma Beat Wave Accelerator
}

\author{
W. P. Leemans, C. E. Clayton, K. A. Marsh, A. Dyson, and C. Joshi \\ Department of Electrical Engineering \\ University of California at Los Angeles \\ 56-125B Engineering IV \\ Los Angeles, CA 90024
}

\section{Abstract}

Tunneling ionization can be thought of as the high intensity, low frequency limit of multi-photon ionization (MPI). Extremely uniform plasmas were produced by the latter process at Rutherford lab for beat wave excitation experiments using a $0.5 \mu \mathrm{m}$ laser. Plasmas with $100 \%$ ionization were produced with densities exceeding $10^{17} \mathrm{~cm}^{-3}$. Our experiment uses a $\mathrm{CO}_{2}$ lascr $\left(\mathrm{I}_{\max } \approx 5 \times 10^{14} \mathrm{~W} / \mathrm{cm}^{2}\right)$ which allows the formation of plasmas via the tunneling process. For our experiments we need plasmas with densities in the range of 5 to $10 \times 10^{16} \mathrm{~cm}^{-3}$. Using Thomson scattering as a diagnostic we have explored the density and temperature regime of tunneling ionization produced plasmas. We find that plasmas with densities up to $10^{16} \mathrm{~cm}^{-3}$ can indeed be produced and that these plasmas are hot. Beyond this density strong refraction of laser radiation occurs due to the radial profile of the plasma. Implications of this work to our Beat Wave Accelerator program will be discussed.

\section{INTRODUCTION}

In plasma accelerator schemes such as laser beat wave, laser wakefield, and electron wakefield accelerators, it is desirable to have long regions of homogeneous plasma at fairly high densities $\left(10^{16} \cdot 10^{18} \mathrm{~cm}^{-3}\right)$. Such plasmas are conventionally produced by electric discharges in gases with a wide variety of discharge geometries. In our experiment on beat wave acceleration, we were forced to abandon dischargeproduced plasmas because the minimum discharge current needed to produce a suitable electron density also produced a magnetic field large enough to prevent our $1.5 \mathrm{MeV}$ electron probing beam from being trapped in the accelerating potential of the plasma beat wave. ${ }^{1}$ But experiments with an intense $0.5 \mu \mathrm{m}$ laser have shown that plasmas with the desired properties could be produced by MPI. ${ }^{2}$ Later, improvements

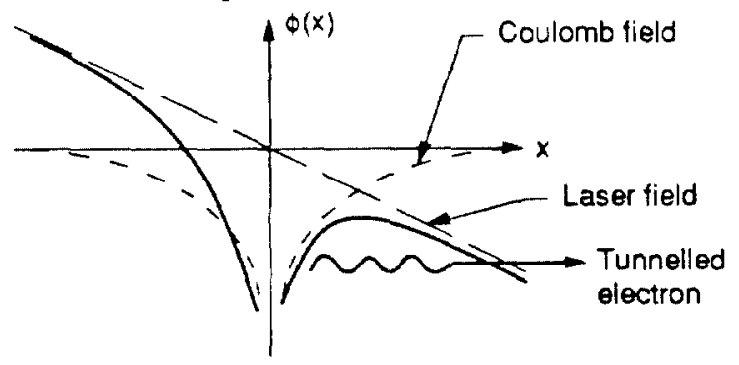

Figure 1: Depiction how an intense external electric field can lead to an electron tunnelling out of the confining potential of an inn.

This work is supported by DOE contract number DEAS03-83-ER40120. in our $10 \mu \mathrm{m} \mathrm{CO} 2$ laser system allowed us to reach intensities where we could produce plasmas by tunneling ionization.

In tunneling ionization, ${ }^{3}$ an external electric field lowers (on one side) the Coulomb potential which binds an electron to the nucleus, as shown in Fig. 1, thereby narrowing the barrier to the outside world. If the field is high enough, the electron has a high probability of tunneling through the barrier where it is free of the nucleus. Its subsequent motion will be composed of the usual quiver motion in the laser field plus a DC drift velocity needed to balance canonical momentum before and after the time of ionization. A landmark experiment by Corkum et al. showed that when ionizing atoms in the single particle regime, one can predict the exact electron distribution functions (that is, the distribution of DC drift velocities) given the laser polarization and pulse shape. From the simple 1-D theory, we expect to produce fully ionized plasmas in the desired density range. For linear polarization, the calculated transverse (longitudinal) electron distribution function resembles a maxwellian with a width of about 50 $(<1) \mathrm{eV}$ while for circular polarization the distribution function should be a "squashed doughnut" with a major radius (transverse to $\mathrm{k}_{0}$ ) of $2.5 \mathrm{KeV}$ and a minor radius of $1 \mathrm{KeV}$ with a longitudinal thickness of only $4 \mathrm{eV}$ ( $k_{0}$ is the wavevector of the incident $\mathrm{CO}_{2}$ laser).

This paper reports on our experiments designed to establish the ionization mechanism and explore the characteristics of this plasma.

\section{THE EXPERIMENT}

In the experiment, a $\mathrm{CO}_{2}$ laser beam (up to $100 \mathrm{~J}, 350$ psec FWHM) is focused into a vacuum chamber containing 0 2 Torr of $\mathrm{H}_{2}, \mathrm{Ar}$, or $\mathrm{He}$ gas. The peak laser intensity in vacuum is around $5 \times 10^{14} \mathrm{~W} / \mathrm{cm}^{2}$. The plasma is produced over the Raleigh length of roughly $2 \mathrm{~cm}$ and is diagnosed by: (a) viewing the laser harmonic emission in the forward direction; (b) X-ray emission from the plasma; (c) collective Thomson scattering of a frequency-doubled YAG probe beam, looking at $2 \mathrm{~K}_{0}$ density fluctuations; and (d) by viewing laser light scattered outside the original cone angle of the beam.

\section{RESULTS AND DISCUSSION}

\section{Harmonics}

The frequency spectrum of the transmitted or forward scattered laser light was found to contain discrete lines at harmonics of the laser frequency. The relative power in these lines are shown in Fig. 2(a) vs harmonic number. The odd harmonics are predicted from the tunneling picture of ionization. ${ }^{5}$ As the laser electric field oscillates at $\omega_{0}$, 
ionization will occur twice per cycle when the electric field reaches its positive or negative extreme. Thus the electron density f increases step-wise in time at $2 \omega_{0}$ (and in space at $\left.2 \mathrm{k}_{\mathrm{o}}\right)$. This second harmonic density modulation $\tilde{n}\left(2 \omega_{0}, 2 \mathrm{k}_{0}\right)$ times the quiver motion $v\left(\omega_{0}, k_{0}\right)$ due to the laser generates currents $\mathrm{J}\left(1 \omega_{0}, l \mathrm{k}_{\mathrm{o}}\right)=-$ evñ, $\mathrm{l}=3,5,7, \ldots$ which radiate the harmonic spectrum. In circular polarized light, the laser electric field does not oscillate in magnitude but only in direction. Thus the density should not be modulated in time and the odd harmonics should go away. This indeed occurs as

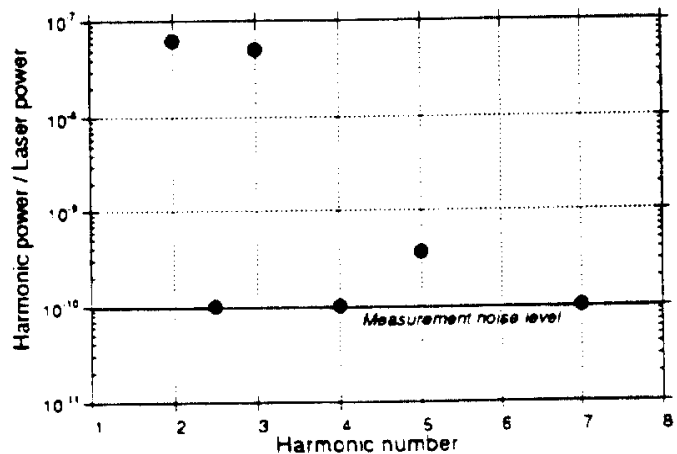

(a)

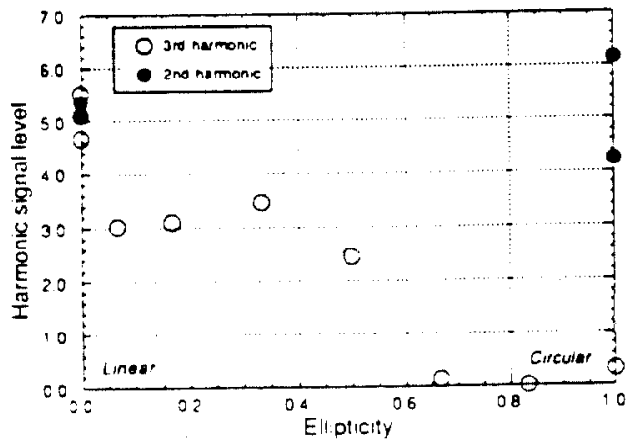

(b)

Figure 2: (a) Normalized power measured in the various laser harmonics. (b) Dependence of the level of the second and third harmonics on the polarization of the laser, changing from linear polarization to circular polarization.

shown in Fig. 2(b) which plots the amplitude of the third harmonic as a function of the ellipticity of the beam. We also observed second harmonic generation and find it to be insensitive to polarization. This harmonic is not predicted from tunneling ionization theory. We believe it is due to the presence of laser light bending through a density gradient and hence a signature of refraction of the laser light. This refraction has serious consequences as will be discussed later.

\section{Temperature}

Apart from harmonics, the other crucial prediction of tunneling ionization theory is that the temperature can be controlled through the polarization of the ionizing laser light. Fig. 3 shows the integrated $X$-ray flux falling on a silicon surface barrier detector filtered to detect about $800 \mathrm{eV}$ photons and above. The three sets of data points are for circular polarization, linear with the detector looking along the electric field, and linear looking transverse to the electric field. A significant difference in the $\mathrm{X}$-ray flux is indeed seen when

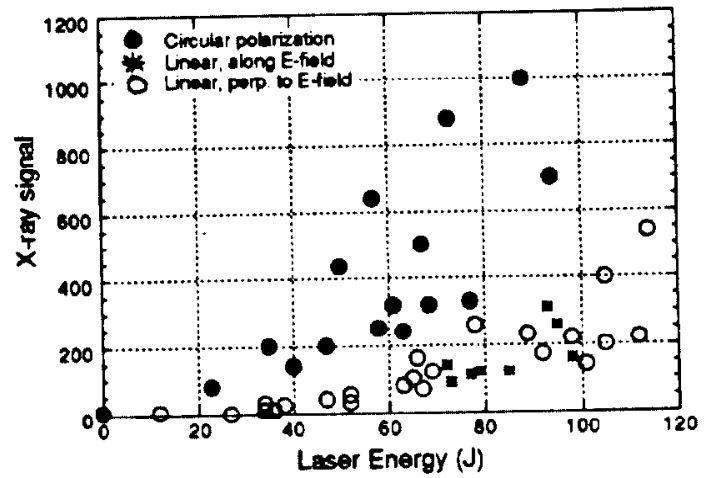

Figure 3: Integrated $\mathrm{X}$-ray flux on a $800+\mathrm{eV}$ detector vs laser energy for three situations: for circular polarization $(\bullet)$, linear polarization with detector viewing perpendicular to the laser electric field $(0)$, and linear polarization with detector viewing along to the laser electric field $\left(^{*}\right)$.

changing the polarization from linear to circular. What is surprising is the presence of any high energy photons in the linear polarization case as we expected a temperature of $<50$ $\mathrm{eV}$. We suspect the existence of an anomalous laser heating mechanism and have seen such evidence in other experiments ${ }^{6}$ as well.

\section{Density}

Our main density diagnostic is based on the detection of electron plasma waves excited by the $\mathrm{C}_{2}$ laser beam. These waves have a frequency $\omega_{\mathrm{BG}} \approx \omega \mathrm{P}\left(1+3 \mathrm{kP}^{2} \lambda_{\mathrm{De}^{2}}\right)^{1 / 2}$ where $\omega_{P}=\left(4 \pi n_{0} e^{2 / m}\right)^{1 / 2}$ is the plasma frequency, $n_{0}$ is the electron density, $k P \approx 2 k_{0}$ is the wavenumber for the plasma waves, and $\lambda_{\mathrm{De}}=\left(\mathrm{T}_{\mathrm{el}} / / 4 \pi \mathrm{n}_{\mathrm{o}} \mathrm{e}^{2}\right)^{1 / 2}$ is the electron Debye length with $T_{e l l}$ the electron temperature parallel to $k_{0}$. This expression is approximately true for $k_{P} \lambda_{D e}<0.5$ or so which should be true for most of our cases. In these cases, $\omega_{\mathrm{BG}}$ is well defined and a spectrum of the electron plasma waves at $k$ $=2 \mathrm{k}_{\mathrm{o}}$ should show an isolated peak at $\omega_{\mathrm{BG}}$. Figure 4 shows a typical spectrum of electron plasma waves obtained by collective Thomson scattering of a visible probe beam from the plasma. The spectrum is time resolved with $10 \mathrm{psec}$ resolution with a streak camera. Instead of the expected narrow feature at a density corresponding to the fully ionized fill gas, we consistently saw a broad spectrum with a maximum frequency shift corresponding to a fairly low density of $1-2 \times$ $10^{16} \mathrm{~cm}^{-3}$. The broadband spectra indicate that $\mathrm{kP} \lambda \mathrm{De}$ is not $<0.5$ but much higher indicating some 2-D heating mechanism unanticipated in the 1-D model of tunneling ionization. More importantly, we found that the maximum frequency shift is proportional to the fill pressure of the vacuum chamber only up to densities around $2 \times 10^{16} \mathrm{~cm}^{-3}$ and is clamped to approximately that value for all higher fill pressures. To verify that the low density measurement is correct, we attempted beatwave excitation of plasma waves using two-frequency illumination. When the beat frequency was chosen to correspond to a density of $8 \times 10^{15} \mathrm{~cm}^{-3}$, we always saw a response in the streaked spectrum. These beatwave satellites can be seen in Fig. 4 at about $8 \AA$ of shift. These exist because the plasma can always respond if the 


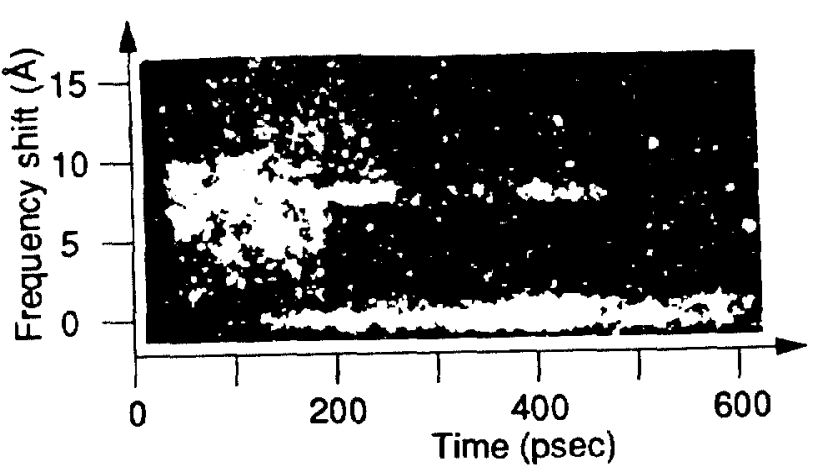

Figure 4: Time-resolved frequency spectrum of $2 \mathbf{k}_{0}$ electron density fluctuations. Frequency increases upwards and time increases to the right. The feature near zero shift is from ion waves and is not important here. The electron plasma wave features range from $0-20 \AA$ shift. The feature at around $8 \AA$ is a beat wave response of the plasma.

density is higher than the beat frequency. However, if we choose a beat frequency corresponding to $10^{17} \mathrm{~cm}^{-3}$ (the desired density), then these satellites were never seen indicating that the density was indeed clamped below $10^{17} \mathrm{~cm}^{-3}$.

\section{Refraction}

From the tunneling ionization theory, the only way that the density cannot be equal to the fill pressure is if the intensity does not increase beyond $5 \times 10^{13} \mathrm{~W} / \mathrm{cm}^{-2}$ (the ionization threshold) for the 20 to 30 psec required to reach full ionization. This would be the case if the laser beam refracts as it begins to ionize at $5 \times 10^{13} \mathrm{~W} / \mathrm{cm}^{-2}$, blowing up the spot size and preventing further ionization. This ionization-induced refraction must occur for gaussian beams where the intensity profile and hence the ionization profile peaks on axis. The plasma then looks like a negative lens to subsequent laser light.

To see whether this refraction is important for our parameters, we measured the amount of light exiting the plasma outside the original cone angle of the beam as a function of fill gas pressure. The result is shown in Fig. 5 for $\mathrm{H}_{2}$ and $\mathrm{Ar}$ gas. In $\mathrm{Ar}$, as we raised the fill pressure from

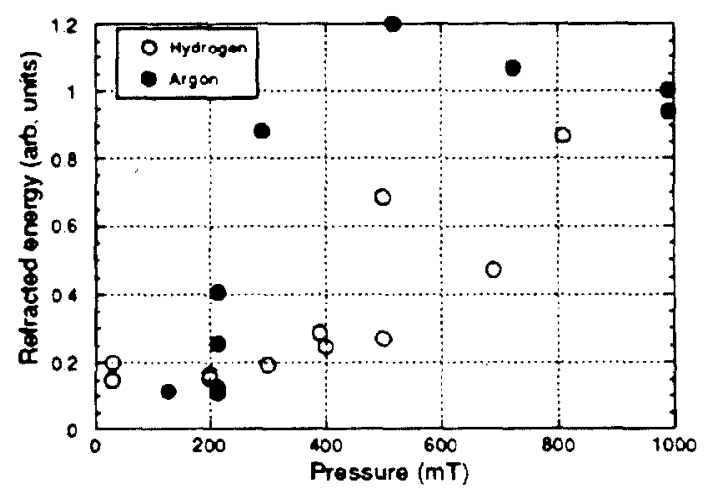

Figure 5: Variation of the energy refracted out of the original cone angle of the laser beam vs fill pressure. Note the sudden onset of refraction in Ar gas beyond about $200 \mathrm{mT}$. vacuum we saw a sudden onset of refraction at around $200 \mathrm{mT}$. This corresponds to a density of around $0.7 \times 10^{16} \mathrm{~cm}^{-3}$. When we repeated the experiment in $\mathrm{H}_{2}$, we noticed that the refraction is a more gradual function of pressure. The difference is due to the different ion masses. On the laser pulse time scale the Ar ions are relatively immobile and the ionization profile is frozen in whereas the $\mathrm{H}_{2}$ ions can move and relax the density gradients in the plasma and thus reduce the defocusing. It therefore seems that refraction is indeed the mechanism limiting our density to $<1-2 \times 10^{16} \mathrm{~cm}^{-3}$.

\section{CONCLUSIONS}

Although multiphoton ionization with $0.5 \mu \mathrm{m}$ laser light was shown to produce large, uniform plasmas at a density of $10^{17}$ $\mathrm{cm}^{-3}$, tunneling ionization with $10 \mu \mathrm{m}$ laser light is limited to densities below about $1-2 \times 10^{16} \mathrm{~cm}^{-3}$. In the $10 \mu \mathrm{m}$ case, the density limit is $0.1-0.2 \%$ of the critical density over about $1 \mathrm{~cm}$ of length. The refraction angle should scale as $\int n_{0}(x) d x$ along the axis of the plasma. Thus, if we can reduce the length of plasma by a factor of 10 we can possibly raise the density a factor of 10 . This is the motivation for the next experiment where we have already installed a $1.5 \mathrm{~mm}$ diam, Mach 8 gas jet for a target rather than a static fill of gas. It seems likely that the laser intensity will reach a high enough intensity on both sides of the gas jet to achieve full ionization across the jet.

\section{REFERENCES}

1. C. Joshi, C. E. Clayton, W. P. Leemans, K. A. Marsh, M. T. Shu, and R. L. Williams, "Beatwave acceleration experiments at UCLA," in Proc. IEEE Particle Accelerator Conf., Chicago, Il, March 1989, pp. 726-730.

2. A. E. Dangor, A. K. L. Dymoke-Bradshaw, A. Dyson, T. Garvey, 1. Mitchell, A. J. Colc, C. N. Danson, C. B. Edwards, and R. G. Evans, "Generation of uniform plasmas for beat wave experiments," IEEE Trans. Plasma Sci., vol. PS-15, pp. 161-166, April 1987.

3. L. V. Keldysh, "Ionization in the field of a strong electromagnetic wave," Sov. Phys. JETP, vol. 20, pp. 1307 14, May 1965.

4. P. B. Corkum, N. H. Burnett and F. Brunel, "Above-threshold ionization in the long-wavelength limit," Phys. Rev. Lett., vol. 62, pp. 1259-62, March 1989.

5. F. Brunel "Harmonic generation due to plasma effects in a gas undergoing multiphoton ionization in the high-intensity limit," J. Opt. Soc. Am. B, vol. 7, pp. 521-26, April 1990.

6. W. P. Leemans, C. E. Clayton, K. A. Marsh, and C. Joshi, "Stimulated compton scattering from pre-formed underdense plasmas," submitted to Phys. Rev. Lett.. 